\title{
Safety, efficacy, and response predictors of anticoagu- lation for the treatment of nonmalignant portal-vein thrombosis in patients with cirrhosis: a propensity score matching analysis
}

\author{
Jung Wha Chung, Gi Hyun Kim, Jong Ho Lee, Kyeong Sam Ok, Eun Sun Jang, Sook-Hyang Jeong, and Jin-Wook Kim
}

Department of Internal Medicine, Seoul National University College of Medicine, Seoul, Korea

Background/Aims: Portal-vein thrombosis (PVT) develops in $10-25 \%$ of cirrhotic patients and may aggravate portal hypertension. There are few data regarding the effects of anticoagulation on nonmalignant PVT in liver cirrhosis. The aim of this study was to elucidate the safety, efficacy, and predictors of response to anticoagulation therapy in cirrhotic patients.

Methods: Patients with liver cirrhosis and nonmalignant PVT were identified by a hospital electronic medical record system (called BESTCARE). Patients with malignant PVT, Budd-Chiari syndrome, underlying primary hematologic disorders, or preexisting extrahepatic thrombosis were excluded from the analysis. Patients were divided into two groups (treatment and nontreatment), and propensity score matching analysis was performed to identify control patients. The sizes of the thrombus and spleen were evaluated using multidetector computed tomography .

Results: Twenty-eight patients were enrolled in this study between 2003 and 2014: 14 patients who received warfarin for nonmalignant PVT and 14 patients who received no anticoagulation. After 112 days of treatment, 11 patients exhibited significantly higher response rates (complete in 6 and partial in 5) compared to the control patients, with decreases in thrombus size of $>30 \%$. Compared to nonresponders, the 11 responders were older, and had a thinner spleen and fewer episodes of previous endoscopic variceal ligations, whereas pretreatment liver function and changes in prothrombin time after anticoagulation did not differ significantly between the two groups. Two patients died after warfarin therapy, but the causes of death were not related to anticoagulation.

Conclusions: Warfarin can be safely administered to cirrhotic patients with nonmalignant PVT. The presence of preexisting portal hypertension is a predictor of nonresponse to anticoagulation. (Clin Mol Hepatol 2014;20:384-391)

Keywords: Portal vein; Venous thrombosis; Liver cirrhosis; Warfarin; Propensity score

\section{INTRODUCTION}

Liver cirrhosis is the end stage of liver disease, and presents various clinical manifestations, including thrombocytopenia, pro- longed prothrombin time (PT), ascites, encephalopathy, and variceal bleeding. As the survival rate of patients with liver cirrhosis has increased over the years, physicians have encountered more complications and complex clinical situations in cases of liver cir-

\section{Abbreviations:}

ALT, alanine transaminase; AST, aspartate transaminase; APRI, ASTto-platelet ratio index; CT, computed tomography; INR, international normalized ratio; PT, prothrombin time; PVT, portal vein thrombosis

\section{Corresponding author : Jin-Wook Kim}

Department of Internal Medicine, Seoul National University Bundang Hospital, 82 Gumi-ro 173 beon-gil, Bundang-gu, Seongnam 463-707, Korea

Tel: +82-31-787-7009, Fax: +82-31-787-4051

E-mail:kimjw@snubh.org 
rhosis. One common problem yet to be solved is thrombosis of the portal vein. Portal vein thrombosis (PVT) occurs at a rate of $5-26 \%$ in different groups of PVT patients, and the occurrence increases as liver disease progresses. ${ }^{1,2}$ It may potentially lead to hepatic decompensation by increasing portal hypertension and the risk of variceal bleeding, and by decreasing liver perfusion., ${ }^{3,4}$ Furthermore, the extension of PVT may increase surgical complexity, which is associated with a high risk of perioperative morbidity and mortality, or exclude patients from transplantation. ${ }^{5}$ Nevertheless, there are no established treatment guidelines or a consensus about PVT at present, and the efficacy of anticoagulation treatment remains unclear. Several control studies have evaluated that anticoagulation might be effective for treating cirrhosis with nonmalignant PVT only. Because only four studies have reported anticoagulation treatment in patients with cirrhosis and PVT, excluding cases of mesenteric venous thrombosis, ${ }^{6-9}$ we sought to evaluate the clinical implications of anticoagulation treatment in patients with cirrhosis and non-malignant PVT.

A propensity score is the probability that a patient receives a particular treatment considering a set of observed covariates. Propensity score matching can be used to reduce selection bias by matching patients based on these covariates, which in this case are the different severities of liver cirrhosis. ${ }^{10,11}$ The present study aimed to assess the efficacy and safety of anticoagulation therapy using warfarin in patients with non-malignant PVT based on the severity of liver cirrhosis. To adjust for the selection bias inherent in retrospective observational studies, propensity score matching was performed. 10,11 The hypothesis of this study was that warfarin therapy would affect the recanalization of non-malignant PVT favorably and safely in patients with liver cirrhosis.

\section{MATERIALS AND METHODS}

\section{Patients}

Patients who developed PVT from April 2003 to June 2014 at Seoul National University Bundang Hospital were considered eligible for the study. Before enrollment, all patients treated with warfarin had their medical records thoroughly reviewed based on our hospital's electronic medical record system (BESTCARE). Patients with liver cirrhosis who were diagnosed with PVT were included in the study. Liver cirrhosis was diagnosed by a clinician on the basis of findings of laboratory and imaging studies, and PVT was confirmed by two distinct radiology specialists using multidetector computed tomography (CT). Patients with malignant PVT, underlying primary hematologic disorders, Budd-Chiari syndrome, membranous obstruction of the inferior vena cava, or pre-existing extrahepatic thrombosis were excluded. Patients who had been lost to follow-up were also excluded from the analysis.

Data of demographics, liver disease etiology, durations and doses of warfarin, history of heavy alcohol drinking, history of gastrointestinal bleeding, and presence of malignancy were collected from the BESTCARE medical record system for all patients. Clinical, laboratory, and radiologic data were collected just before the initiation and cessation of anticoagulation treatment. Laboratory data, such as aspartate transaminase (AST) level, alanine transaminase (ALT) level, bilirubin level, platelet count, PT, and albumin and creatinine levels at the time of PVT diagnosis were collected, as well as those at the last follow-up. Accompanying ascites, longest diameter of thrombus, and thickness of the spleen were evaluated using abdominopelvic CT. SI was calculated by multiplying longest width, thickness of spleen in coronal CT image and length of spleen in sagittal CT image. (Splenic Index [SI]) = Splenic width $\times$ splenic thickness $\times$ splenic length $\left[\mathrm{cm}^{3}\right]$ )

Initiation of warfarin treatment was decided by a clinician, and started as soon as possible whenever definite non-malignant thrombosis was diagnosed. The cessation of treatment was decided after evaluation of the effects of anticoagulation therapy on thrombosis within at least three months follow-up using $C T$, and the follow-up imaging studies were performed depending on underlying disease. The study was approved by the internal review board and ethics committee of our institution.

\section{Definition}

Non-malignant thrombosis was defined as the absence of malignant thrombosis features, such as an irregular expansile pattern of growth, suspicious arterial enhancement and ballooning, or direct invasion of the adjacent portal vein by malignant tumor. Heavy alcohol use was classically defined as a daily alcohol intake of more than $80 \mathrm{~g}$ in men and more than $40 \mathrm{~g}$ in women. The longest diameter of the main thrombus was measured from one representative transverse $\mathrm{CT}$ image. The time between the first detection of thrombosis by imaging studies and the introduction of anticoagulation therapy was identified. The duration of thrombosis was defined as the time between first detection and the initiation of anticoagulation therapy. The response to warfarin or the natural course of the thrombus was evaluated based on changes in diameter at scheduled follow-up assessments. Complete resolution 
was defined as disappearance of all evidence of thrombosis, as determined by transverse CT images. Partial resolution was defined as at least a 30\% reduction in the long diameter of the main thrombus, that is, more than a $50 \%$ decrease in cross-sectional area without evidence of the appearance of new thrombi. Patients with complete or partial resolution were regarded as responders in the warfarin treatment group. Non-response was defined as a decrease in thrombus size that did not qualify for partial resolution, or as a progression of thrombosis or the appearance of new thrombus.

\section{Statistical analyses}

To adjust for potential confounding resulting from significant differences in baseline liver function, propensity score matching was used to select the patients in the untreated control group. The propensity score of each patient in the warfarin treatment group was calculated using the Child-Pugh score, AST-to-platelet ratio index (APRI) and diameter of thrombus by software package $R$, version 3.1.1 (R Development Core Team, 2014). Matching was performed by selecting untreated patients and looking for a control patient with the nearest logit-transformed propensity score. The balance of matching variables was assessed by formal statistical comparison: Kolmogorov-Smirnov test for those continuous variables.

To compare continuous numeric variables between the groups for descriptive analysis, a Mann-Whitney test was performed for all numerical variables. Categorical variables were analyzed by the chi-square test or Fisher's exact test. Fisher's exact test was used to compare the response of anticoagulation between the groups using SPSS software, versions 20.0 (SPSS, Inc., Chicago, IL, USA). A $P$-value of $<0.05$ was considered statistically significant.

\section{RESULTS}

\section{Characteristics of the study population}

In the warfarin treatment group, six patients met exclusion criteria, and one patient was lost to follow-up. Eventually, fourteen cirrhotic patients with non-malignant PVT were enrolled in the warfarin treatment group. Fourteen of 72 eligible untreated patients with non-malignant thrombosis, who were individually matched against the warfarin treatment group using propensity score matching, were enrolled in the control group. A total of 28 pa- tients were finally analyzed (Fig. 1). The relevant baseline characteristics of the enrolled patients are showed in Table 1. After propensity matching, differences of the initial spleen thickness, the SI, and the prevalence of prior HCC treatment between the groups decreased. The calculated SI of patients in the warfarin treatment group was greater than those of patients in the control group, but this difference was not statistically significant.

Thrombosis was detected only in one portal vein branch (i.e. the main portal vein) in half of both groups, but about $35 \%$ of cirrhotic patients in the warfarin treatment group had thrombi in two or more portal veins (Table 2). The mean longest diameter of the main thrombus was $25.9 \mathrm{~mm}$ in the warfarin treatment group, and $24.7 \mathrm{~mm}$ in the control group.

\section{Response of portal vein thrombosis to warfarin treatment}

Duration of warfarin treatment was $112 \pm 62$ days (mean \pm standard deviation) and a mean dose $2.7 \mathrm{mg} /$ day was used. PT (International normalized ratio [INR]) in the warfarin treatment group was $1.90 \pm 0.41$, which showed no significant difference to that in the control group $(1.43 \pm 0.18)$. In the warfarin treatment group, 11 of $14(78.6 \%)$ patients showed thrombus resolution (complete resolution in six; partial resolution in five), whereas only five of 14 patients (35.7\%) showed thrombus resolution in the control group ( $P=0.022$, Table 3 ). A case of complete PVT resolution after warfarin therapy is shown in Figure 2. Without anticoagulation therapy, three patients (21.4\%) showed complete resolution, and

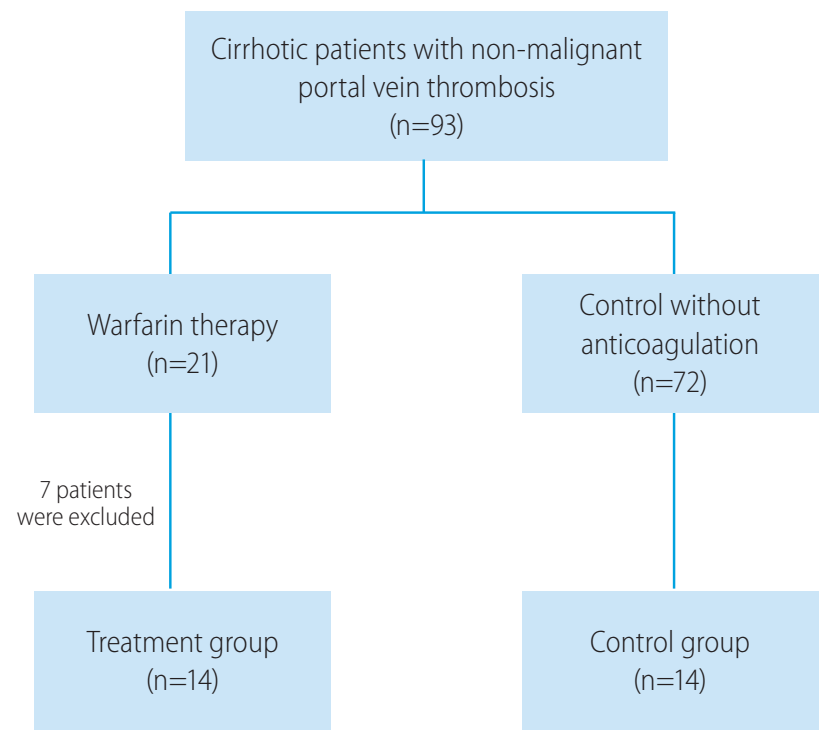

Figure 1. Patient enrollment in the present study. 
Table 1. Patient characteristics

\begin{tabular}{|c|c|c|c|c|c|}
\hline Variable & $\begin{array}{c}\text { Warfarin } \\
\text { therapy } \\
(n=14)\end{array}$ & $\begin{array}{l}\text { Control before } \\
\text { matching } \\
(n=72)\end{array}$ & $P$-value & $\begin{array}{c}\text { Control } \\
(n=14)\end{array}$ & $\boldsymbol{P}$-value \\
\hline Age, years & $59.4 \pm 12.0$ & $61.1 \pm 11.1$ & 0.516 & $58.7 \pm 13.2$ & 0.874 \\
\hline Male sex, no. (\%) & $10(71.4)$ & $57(79.2)$ & 0.500 & $11(78.6)$ & 1.000 \\
\hline \multicolumn{6}{|l|}{ Cause of cirrhosis, no } \\
\hline Alcohol / HBV / Others & $3 / 10 / 1$ & $18 / 48 / 6$ & 0.957 & $4 / 9 / 1$ & 0.798 \\
\hline Child-Pugh classification, A / B / C & $6 / 8 / 0$ & $37 / 32 / 3$ & 0.792 & $7 / 6 / 1$ & 1.000 \\
\hline APRI score & $2.05 \pm 1.1$ & $1.79 \pm 1.7$ & 0.152 & $1.71 \pm 1.1$ & 0.376 \\
\hline PT (INR) & $1.38 \pm 0.28$ & $1.32 \pm 0.20$ & 0.206 & $1.43 \pm 0.18$ & 0.571 \\
\hline Initial spleen thickness, cm & $6.7 \pm 1.4$ & $5.9 \pm 6.6$ & 0.001 & $5.9 \pm 1.5$ & 0.085 \\
\hline Splenic index (SI), $/ \mathrm{cm}^{3 *}$ & $2218 \pm 1301$ & $1485 \pm 1745$ & 0.004 & $1659 \pm 1170$ & 0.114 \\
\hline Mean follow-up duration, days & $115 \pm 92$ & - & - & $108 \pm 90$ & 0.603 \\
\hline Underlying hepatocellular carcinoma, no. (\%) & $8(57.1)$ & $48(66.7)$ & 0.547 & $8(57.1)$ & 1.000 \\
\hline Prior HCC treatment, no. (\%) & $3(21.4)$ & $40(55.6)$ & 0.019 & $5(35.7)$ & 0.678 \\
\hline Varix ligation before presence of PVT, no. (\%) & $4(28.6)$ & $28(38.9)$ & 0.465 & $5(35.7)$ & 1.000 \\
\hline
\end{tabular}

Continuous variables are expressed as mean \pm standard deviation.

APRI, AST-to-platelet ratio index; HCC, hepatocellular carcinoma; INR, international normalized ratio; PT, prothrombin time; PVT, portal vein thrombosis.

*Splenic Index (SI)=Splenic width $\times$ splenic thickness $\times$ splenic length $\left(\mathrm{cm}^{3}\right)$

Table 2. Characteristics of portal-vein thrombosis

\begin{tabular}{|c|c|c|c|}
\hline Variable & $\begin{array}{l}\text { Warfarin therapy } \\
\qquad(n=14)\end{array}$ & Control $(n=14)$ & $P$-value \\
\hline \multicolumn{4}{|l|}{ Vessel with thrombosis, no (\%) } \\
\hline Main portal vein & $9(64.3)$ & $9(64.3)$ & \\
\hline Right portal vein & $5(35.7)$ & $7(50.0)$ & \\
\hline Left portal vein & $2(14.3)$ & $4(40.0)$ & \\
\hline Superior mesenteric vein & $3(21.4)$ & $2(14.3)$ & \\
\hline Number of vessels with thrombosis, 1 / 2 / 3 (\%) & $\begin{array}{c}9 / 3 / 2 \\
(64.3 / 21.4 / 14.3)\end{array}$ & $\begin{array}{c}8 / 4 / 2 \\
(57.1 / 28.6 / 14.3)\end{array}$ & 0.800 \\
\hline Longest diameter in transverse section, $\mathrm{mm}$ & $25.9 \pm 8.9$ & $24.7 \pm 7.1$ & 0.734 \\
\hline
\end{tabular}

Continuous variables are expressed as mean \pm standard deviation.

two patients (14.3\%) showed partial resolution of the main thrombus in the control group. Another three patients (21.4\%), however, had thrombi that increased sized of the main thrombus or newly developed thrombus in the control group, compared to only one patient in warfarin treatment group. Response of thrombus, but, showed no significance after Kaplan-Meier analysis $(P=0.156$, by generalized Wilcoxon)

A 4-fold increase of AST and ALT was noted as a warfarin-related complication at 72 days after the initiation of treatment in only one patient, who as a result had to stop warfarin treatment within 2.5 months. Two patients expired in warfarin treatment group after the cessation of warfarin therapy; one patient expired two years after treatment due to septic shock and the other patient expired one month after cessation of treatment due to chemoembolization-induced renal failure. On the other hand, four control patients expired during follow-up because of esophageal variceal bleeding, hepatorenal syndrome, septic shock and subarachnoid hemorrhage, respectively. In the warfarin treatment group, bleeding or any other complications were not observed, and no other patients had to have their therapy interrupted.

\section{Predictors of thrombus resolution}

Among patients in the warfarin treatment group, responders 

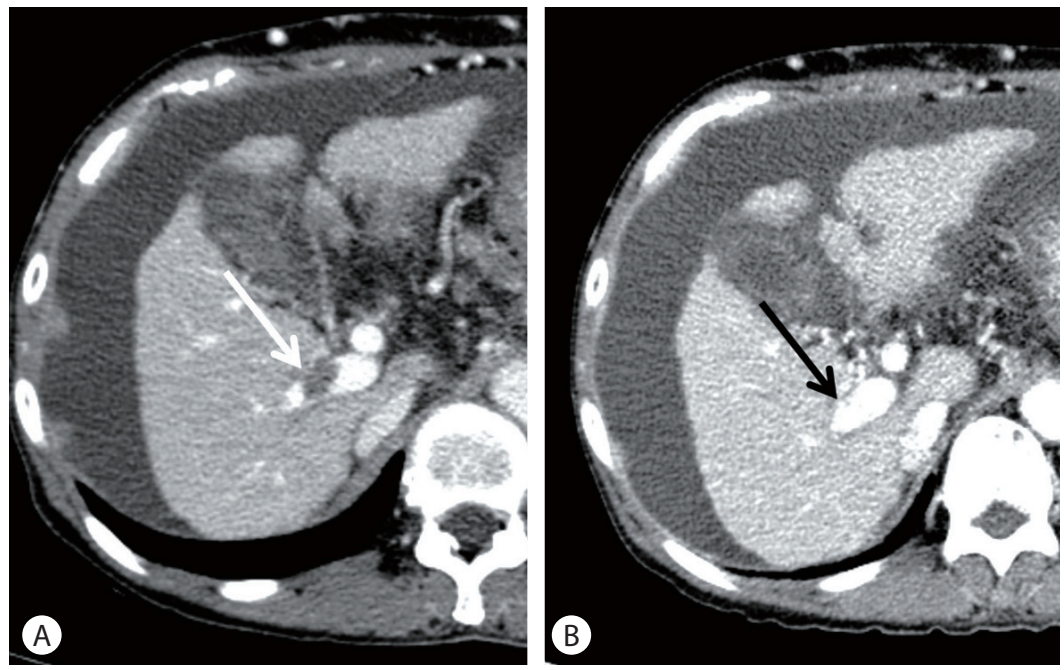

Figure 2. Complete resolution of portal-vein thrombosis after warfarin therapy. The patient was a 61-year old woman who achieved recanalization after warfarin therapy. (A) Transverse computed tomography scan obtained before anticoagulation showing a portal-vein thrombus in the right portal vein of the liver (white arrow). (B) Follow-up computed tomography scan obtained 1.5 months after warfarin therapy. Note the absence of a thrombus in the right portal vein (black arrow indicates the position of the thrombus pretreatment; white arrow in A).

Table 3. Effect of warfarin therapy on a portal-vein thrombus

\begin{tabular}{lccc}
\hline Response & $\begin{array}{c}\text { Warfarin treatment } \\
\text { group }(\mathbf{n}=\mathbf{1 4})\end{array}$ & $\begin{array}{c}\text { Control group } \\
(\mathbf{n}=\mathbf{1 4})\end{array}$ & $\begin{array}{c}\boldsymbol{P} \text {-value } \\
\text { Thrombus resolution }\end{array}$ \\
Non-response of thrombus & 11 & 5 & 0.022 \\
\hline
\end{tabular}

$P$-value derived from Fisher's exact test.

Table 4. Predictors of portal-vein thrombus resolution by anticoagulation (warfarin)

\begin{tabular}{|c|c|c|c|}
\hline Variable & $\begin{array}{l}\text { Thrombus resolution } \\
\qquad(\mathrm{n}=11)\end{array}$ & $\begin{array}{l}\text { Non-response } \\
\quad(n=3)\end{array}$ & $\boldsymbol{P}$-value \\
\hline Age, years & $61.9 \pm 12.1$ & $50.0 \pm 6.0$ & 0.170 \\
\hline Male sex, no. (\%) & $8(72.7)$ & $2(66.7)$ & 1.000 \\
\hline Heavy alcohol drinking, no. (\%) & $2(18.2)$ & $2(66.7)$ & 0.176 \\
\hline Duration of anticoagulation, days & $98 \pm 63$ & $166 \pm 31$ & 0.060 \\
\hline Thrombus duration > 6 months, no. (\%) & $0(0)$ & $2(66.7)$ & 0.033 \\
\hline Child-Pugh class B, no. (\%) & $6(54.5)$ & $2(66.7)$ & 1.000 \\
\hline Underlying HCC, no. (\%) & $6(54.5)$ & $2(66.7)$ & 1.000 \\
\hline History of previous EVL, no. (\%) & $2(18.2)$ & $2(66.7)$ & 0.176 \\
\hline APRI score & $1.96 \pm 1.2$ & $2.4 \pm 0.8$ & 0.555 \\
\hline PT (INR) & $1.35 \pm 0.31$ & $1.47 \pm 0.06$ & 0.291 \\
\hline$\triangle I N R$ during treatment & $0.59 \pm 0.49$ & $0.28 \pm 0.14$ & 0.368 \\
\hline WBC count, $/ \mathrm{mm}^{3}$ & $3935 \pm 1743$ & $1680 \pm 869$ & 0.060 \\
\hline Platelet count, $\times \mathrm{K} / \mathrm{mm}^{3}$ & $71 \pm 31$ & $37 \pm 10$ & 0.060 \\
\hline Spleen thickness, before treatment, $\mathrm{cm}$ & $6.3 \pm 1.2$ & $8.4 \pm 0.1$ & 0.038 \\
\hline Splenic index $(\mathrm{SI}), / \mathrm{cm}^{3}$ & $1882 \pm 1153$ & $3453 \pm 1200$ & 0.060 \\
\hline
\end{tabular}

Continuous variables are expressed as mean \pm standard deviation.

"Time between thrombus detection and initiation of anticoagulation.

APRI, AST-to-platelet ratio index; EVL, endoscopic variceal ligation; HCC, hepatocellular carcinoma; INR, international normalized ratio; PT, prothrombin time; PVT, portal vein thrombosis; WBC, white blood cell.

Splenic Index $(\mathrm{SI})=$ Splenic width $\times$ splenic thickness $\times$ splenic length $\left(\mathrm{cm}^{3}\right)$. 
$(n=11,78.6 \%)$ were analyzed with non-responders $(n=3)$ (Table 4). The proportion of patients with a duration of PVT detection to treatment initiation of more than 6 month was much higher in non-responders than in responders ( $66.7 \%$ vs. $0 \%, P=0.033)$. Responders were more likely to have smaller main thrombi $(24.4 \mathrm{~mm}$ vs. $31.7 \mathrm{~mm}, P=0.208)$, lower prevalence of heavy alcohol drinking $(18.2 \%$ vs. $66.7 \%, P=0.176)$, higher white blood cell and platelet counts, and less thickness of the spleen, even if they were found to have received a shorter duration of anticoagulation therapy and were older than non-responders. Spleen thickness at the beginning of the study was the only significant predictor of response $(P=0.038)$.

\section{DISCUSSION}

In this study, we used propensity score matching to assess cirrhotic patients with non-malignant PVT. Recently, propensity score matching has been used in retrospective cohort studies in the field of hepatology to minimize selection bias. ${ }^{12,13}$ After propensity score matching, the baseline characteristics were more accurately matched between warfarin treatment group and control group patients.

Anticoagulation treatment allowed remarkable resolution of thrombosis, although most of the patients with ascites or gastroesophageal varices were generally considered to have decompensated cirrhosis. The number of patients with partial or complete recanalization was significantly higher in the warfarin treatment group (11/14) than in the control group (5/14). The longest diameter of the main thrombus and the proportion of patients with thrombi in more than two vessels, were similar in two groups, but no patients in the warfarin treatment group developed additional PVT lesions while receiving warfarin, compared to three patients in control group who did.

There are limited data demonstrating whether anticoagulation treatment is effective and safe, though it has shown promise in several studies to date. A $50 \%$ rate of patients with portal vein recanalization was reported with low molecular weight heparin in 38 patients with cirrhosis with an episode of non-severe variceal bleeding. ${ }^{14}$ Another study reported complete resolution in $75 \%$ of 39 patients with enoxaparin for $6-17$ months. ${ }^{6}$ A recent study of 55 patients with PVT and cirrhosis reported a $60 \%$ response rate. ${ }^{7}$ Given that the mean warfarin treatment duration was 111.9 days, it is noteworthy that the present study showed thrombus resolution in $78.6 \%$ of patients. Overall, $64 \%$ of patients (9/14) were characterized by a history of malignancy such as hepatocellular carcinoma and colon cancer, which is a well-established risk factor for thrombosis, while the populations of previous studies contained none and two patients with hepatocellular carcinoma respectively. In contrast to previous studies, warfarin was chosen to treat PVT in the present study, and the difference in PT (INR) during the treatment period was only $0.52 \pm 0.45$.

Liver cirrhosis, especially in patients with an advanced stage of the disease, ${ }^{15}$ is no longer considered to be a hypocoagulable state. In fact, a hypercoagulable state is present in patients with liver cirrhosis because levels of liver-derived anticoagulant factors are usually decreased more than those of procoagulant factors. Consequently, patients with liver cirrhosis have a high relative risk of thrombosis. ${ }^{15,16}$ The INR value in liver disease is suspected to overestimate bleeding risk and the international sensitivity index as determined by plasma from patients on vitamin $\mathrm{K}$ antagonists, ${ }^{17}$ which allowed no adjustment of reduction in anticoagulant proteins. ${ }^{18}$ This might explain the achievement of warfarin anticoagulation with lower INR level on thrombosis in present study. However, the hemostatic balance in cirrhosis can be easily dysregulated in certain conditions. ${ }^{19}$ One study reported that anticoagulation with enoxaparin in patients with cirrhosis caused no serious side effects, including bleeding, during 6-17 months of treatment. ${ }^{6}$ In the present study, no significant bleeding events occurred, but early termination due to the elevation of liver enzymes did. Another study showed bleeding complications in 9\% (5/55) cirrhosis patients during a 12 month period. A platelet count of $<50 \times 10^{9} / \mathrm{L}$ was the only factor significantly associated with a higher risk of bleeding complications. ${ }^{7}$ Once variceal bleeding occurs, it is difficult to control in patients with cirrhosis and PVT, and the 1-year survival rate decreases to $61-86 \% .{ }^{20}$ Prophylaxis is therefore important before starting anticoagulation treatment. It must be taken into account that there are concerns about the difficulty of performing hemostasis because of severe bleeding complications, especially in patients with advanced cirrhosis. Controversy still surrounds the efficacy as well as the safety of anticoagulation, and further research regarding these issues should be performed.

The present study identified factors related to a higher probability of resolution. Between responders and non-responders, there were significant differences in the duration between thrombus detection and the initiation of warfarin therapy, and in pre-treatment thickness of the spleen, suggesting that these might be predictors of response to anticoagulation therapy. One study has described these predictors of response previously. Delgado et al. reported that early initiation of anticoagulation therapy in the first 2 weeks 
after the detection of thrombosis was the only factor significantly associated with recanalization, but did not investigate the parameters related to the spleen. The study population was enrolled within 6 months of thrombosis detection during scheduled imaging studies, and both studies suggest the importance of early PVT detection and initiation of anticoagulation in the context of the irreversible progress of PVT with chronicity.

The main limitation of this study was a small study population. Propensity score matching analysis was performed to select the control group, and allowed selection bias to be minimized. ${ }^{4}$ Additionally, PVT may progress or resolve spontaneously, and it is possible that the size of thrombi appears to be modified by warfarin because of a favorable course of non-malignant PVT in cirrhotic patients. One study reported that partial PVT progressed in $48 \%$, improved in $45 \%$, and remained the same in $7 \%$ of 42 patients with cirrhosis. ${ }^{21}$ In the present study, PVT progression was seen in only two $(18.2 \%)$ patients, no change was seen in six patients $(54.5 \%)$, and three patients were lost to follow-up 252 days after the cessation of warfarin therapy. The non-response group included one patient with no change and two patients who were lost to follow-up. The natural course of non-malignant PVT is not yet clear. Due to the short duration of follow-up, improvements in PVT-associated morbidity and mortality rates could not be elucidated in this study. Villa et al. demonstrated in a recent a prospective study that there was significantly less frequent liver decompensation in patients given enoxaparin (11.7\%) for 12 months than in control patients (59.4\%). ${ }^{8}$ In the present study, the proportion of patients who received endoscopic variceal ligation after the occurrence of PVT was higher in the non-response group than in the response group, although this difference was not statistically significant ( $66.7 \%$ vs. $36.4 \%)$.

Currently, there are a number of unresolved issues concerning the management of PVT. In conclusion, warfarin therapy might result in the resolution of non-malignant PVT effectively and safely in patients with liver cirrhosis. Patients with a period between first thrombus detection and the initiation of warfarin therapy of less than six months, and those with less splenic thickness, could have greater responses to anticoagulation therapy. This suggests that the age of non-malignant PVT, and the severity of portal hypertension, might be crucial predictors of the effect of warfarin therapy on non-malignant PVT. Further study is needed to verify the benefits of anticoagulation for non-malignant PVT in patients with cirrhosis. Furthermore, establishing practical guidelines based on reliable evidence is also needed.

\section{Conflicts of Interest}

No potential conflicts of interest were disclosed.

\section{REFERENCES}

1. Boyer TD, Habib S. Portal vein thrombosis in patients with cirrhosis. Clinical Liver Disease 2014;3:111-113.

2. Aqel BA. Vascular Diseases of the Liver. In: Practical Gastroenterology and Hepatology: Liver and Biliary Disease: Wiley-Blackwell, 2010:261-274.

3. Garcia-Pagan JC, Hernandez-Guerra M, Bosch J. Extrahepatic portal vein thrombosis. Semin Liver Dis 2008;28:282-292.

4. DeLeve LD, Valla DC, Garcia-Tsao G. Vascular disorders of the liver. Hepatology 2009:49:1729-1764.

5. Englesbe MJ, Schaubel DE, Cai S, Guidinger MK, Merion RM. Portal vein thrombosis and liver transplant survival benefit. Liver Transpl 2010;16:999-1005.

6. Amitrano L, Guardascione MA, Menchise A, Martino R, Scaglione M, Giovine $S$, et al. Safety and efficacy of anticoagulation therapy with low molecular weight heparin for portal vein thrombosis in patients with liver cirrhosis. J Clin Gastroenterol 2010;44:448-451.

7. Delgado MG, Seijo S, Yepes I, Achecar L, Catalina MV, GarciaCriado $A$, et al. Efficacy and safety of anticoagulation on patients with cirrhosis and portal vein thrombosis. Clin Gastroenterol Hepatol 2012;10:776-783.

8. Villa $E$, Cammà C, Marietta M, Luongo M, Critelli $R$, Colopi $S$, et al. Enoxaparin prevents portal vein thrombosis and liver decompensation in patients with advanced cirrhosis. Gastroenterology 2012;143:1253-1260.e1-4.

9. Werner KT, Sando S, Carey EJ, Vargas HE, Byrne TJ, Douglas DD, et al. Portal vein thrombosis in patients with end stage liver disease awaiting liver transplantation: outcome of anticoagulation. Dig Dis Sci 2013;58:1776-1780.

10. D'Agostino RB, Jr. Propensity score methods for bias reduction in the comparison of a treatment to a non-randomized control group. Stat Med 1998;17:2265-2281.

11. Rubin DB. Estimating causal effects from large data sets using propensity scores. Ann Intern Med 1997;127:757-763.

12. Hosaka T, Suzuki F, Kobayashi M, Seko Y, Kawamura Y, Sezaki H, et al. Long-term entecavir treatment reduces hepatocellular carcinoma incidence in patients with hepatitis B virus infection. Hepatology 2013;58:98-107.

13. Lim YS, Han S, Heo NY, Shim JH, Lee HC, Suh DJ. Mortality, Liver Transplantation, and Hepatocellular Carcinoma Among Patients With Chronic Hepatitis B Treated With Entecavir vs Lamivudine. Gastroenterology 2014;147:152-161. 
14. Senzolo M, Ferronato C, Burra P, Sartori MT. Anticoagulation for portal vein thrombosis in cirrhotic patients should be always considered. Intern Emerg Med 2009;4:161-162; author reply 163-164.

15. Tripodi A, Salerno F, Chantarangkul V, Clerici M, Cazzaniga M, Primignani $M$, et al. Evidence of normal thrombin generation in cirrhosis despite abnormal conventional coagulation tests. Hepatology 2005;41:553-558.

16. Søgaard KK, Horváth-Puhó E, Grønbaek H, Jepsen P, Vilstrup $H$, Sørensen HT. Risk of venous thromboembolism in patients with liver disease: a nationwide population-based case-control study. Am J Gastroenterol 2009;104:96-101.

17. Arjal R, Trotter JF. International normalized ratio of prothrombin time in the model for end-stage liver disease score: an unreliable mea- sure. Clin Liver Dis 2009;13:67-71.

18. Tripodi A. Tests of coagulation in liver disease. Clin Liver Dis 2009;13:55-61.

19. Ben-Ari Z, Osman E, Hutton RA, Burroughs AK. Disseminated intravascular coagulation in liver cirrhosis: fact or fiction? Am J Gastroenterol 1999;94:2977-2982.

20. García-Pagán JC, Caca K, Bureau C, Laleman W, Appenrodt B, Luca A, et al. Early use of TIPS in patients with cirrhosis and variceal bleeding. N Engl J Med 2010;362:2370-2379.

21. Luca A, Caruso S, Milazzo M, Marrone G, Mamone G, Crinò F, et al. Natural course of extrahepatic nonmalignant Partial Portal Vein Thrombosis in Patients with cirrhosis. Radiology 2012;265:124-132. 